\title{
Large resistivity change and phase transition in the antiferromagnetic semiconductors LiMnAs and LaOMnAs
}

\author{
A. Beleanu, J. Kiss, G. Kreiner, C. Köhler, L. Müchler, W. Schnelle, U. Burkhardt, S. Chadov, S. Medvediev, \\ D. Ebke, and C. Felser* \\ Max-Planck-Institut für Chemische Physik fester Stoffe, Dresden, Germany \\ G. Cordier and B. Albert \\ Technical University of Darmstadt, Eduard-Zintl-Institute of Inorganic and Physical Chemistry, Darmstadt, Germany

A. Hoser
Helmholtz Zentrum Berlin für Materialien und Energie GmbH, Berlin, Germany \\ F. Bernardi \\ Departamento de Fisica, Instituto de Fisica, Universidade Federal do Rio Grande do Sul (UFRGS), Porto Alegre, RS, Brazil
}

T. I. Larkin, D. Pröpper, A. V. Boris, and B. Keimer

Max-Planck-Institut für Festkörperforschung, Heisenbergstraße 1, D-70569 Stuttgart, Germany

(Received 30 July 2013; revised manuscript received 5 November 2013; published 27 November 2013)

\begin{abstract}
Antiferromagnetic semiconductors are new alternative materials for spintronic applications and spin valves. In this work, we report a detailed investigation of two antiferromagnetic semiconductors $A \mathrm{MnAs}(A=\mathrm{Li}$, $\mathrm{LaO}$ ), which are isostructural to the well-known LiFeAs and LaOFeAs superconductors. Here we present a comparison between the structural, magnetic, and electronic properties of LiMnAs, LaOMnAs, and related materials. Interestingly, both LiMnAs and LaOMnAs show a variation in resistivity with more than five orders of magnitude, making them particularly suitable for use in future electronic devices. Neutron and $\mathrm{x}$-ray diffraction measurements on LiMnAs show a magnetic phase transition corresponding to the Néel temperature of $373.8 \mathrm{~K}$, and a structural transition from the tetragonal to the cubic phase at $768 \mathrm{~K}$. These experimental results are supported by density functional theory calculations.
\end{abstract}

DOI: 10.1103/PhysRevB.88.184429

PACS number(s): 75.50.Pp, 61.05.fm, 72.15.Eb, 74.70.Xa

\section{INTRODUCTION}

The idea of using semiconductors in spintronic devices has stimulated a new field of research. This is because using not only the charge of the electron but also its spin to store and manipulate information has led to a new paradigm in condensed matter research to overcome the limitations set by Moore's law. ${ }^{1}$ Unfortunately, semiconductivity and ferromagnetism above room temperature are not compatible. Presently, to circumvent this issue, diluted magnetic semiconductors such as $\mathrm{GaMnAs}^{2,3}$ and LiZnMnAs ${ }^{4,5}$ are used. Recently, the use of antiferromagnetic (AFM) semiconductors has been proposed, because they have a higher magnetic ordering temperature compared to diluted magnetic semiconductors. One promising candidate material for new spintronic applications is LiMnAs, given its suitable band gap and high magnetic moment per Mn atom. ${ }^{6}$ It was demonstrated that epitaxially grown LiMnAs thin films also show semiconducting behavior, ${ }^{6}$ and their AFM ordering has been confirmed ${ }^{6-8}$ as well. Among the family of structurally related compounds, for LaOMnAs and NdOMnAs giant magnetoresistance has been observed, ${ }^{9,10}$ and in $\mathrm{NdO}_{0.95} \mathrm{~F}_{0.05} \mathrm{MnAs}$ very recently colossal magnetoresistance has been measured. ${ }^{11}$ Thus, LiMnAs, LaOMnAs, and related materials allow for the study and development of new AFM semiconductors, and the compounds are also interesting from a purely academic viewpoint. CuMnAs ${ }^{12}$ has also been suggested as an alternative semiconducting AFM material; however, this compound shows semimetallic behavior in contrast to the desired semiconducting properties. Still, the compounds with the general formula $A \mathrm{Mn} X$, where $A=\mathrm{Li}, \mathrm{Ni}, \mathrm{Cu}$, or $\mathrm{LaO}$ and $X=\mathrm{As}$ or $\mathrm{Sb}$, offer a good starting point for the development of novel semiconducting magnetic materials for applications in devices such as single-electron transistors. ${ }^{13}$

In the ground state, both LiMnAs and LaOMnAs show AFM insulating behavior. Chemically, LiMnAs consists of ionic $\mathrm{Li}^{+}$and tetrahedral [MnAs] ${ }^{-}$layers (see Fig. 1), with $\mathrm{Mn}^{2+}$ in a $d^{5}$ and $\mathrm{As}^{3-}$ in a nominal $s^{2} p^{6}$ closed shell configuration. LiMnAs is isostructural to LiFeAs superconductor. ${ }^{14}$ Structurally, LaOMnAs is closely related to LiMnAs (see Fig. 1) and to the LaOFeAs layered superconductor. In comparison to LiMnAs, where the Mn moments are ordered antiferromagnetically both in the $a b \mathrm{Mn}$ planes and between the Mn planes stacked along the $c$ axis, ${ }^{15}$ in the case of LaOMnAs, the moments are aligned antiferromagnetically in the $a b$ plane but ferromagnetically along $c .^{9}$

Interestingly, in contrast to the expected $5 \mu_{\mathrm{B}}$ of the $S=5 / 2$ $\mathrm{Mn}^{2+}$ ion, neutron scattering data on LiMnAs and LaOMnAs suggest a magnetic moment of approximately 3.4 to $3.8 \mu_{\mathrm{B}}$, which is close to the magnetic moment of $4 \mu_{\mathrm{B}}$ for an $S=2$ $\mathrm{Mn}^{3+}$ ion. This closely resembles the simple rules and general observation made regarding Heusler compounds, ${ }^{16}$ stating that Mn tends to have a large localized magnetic moment with an oxidation state of 3 (in the literature referred to as the Kübler's rule ${ }^{17}$ ) in a nominal $d^{4}$ configuration. The magnetism 


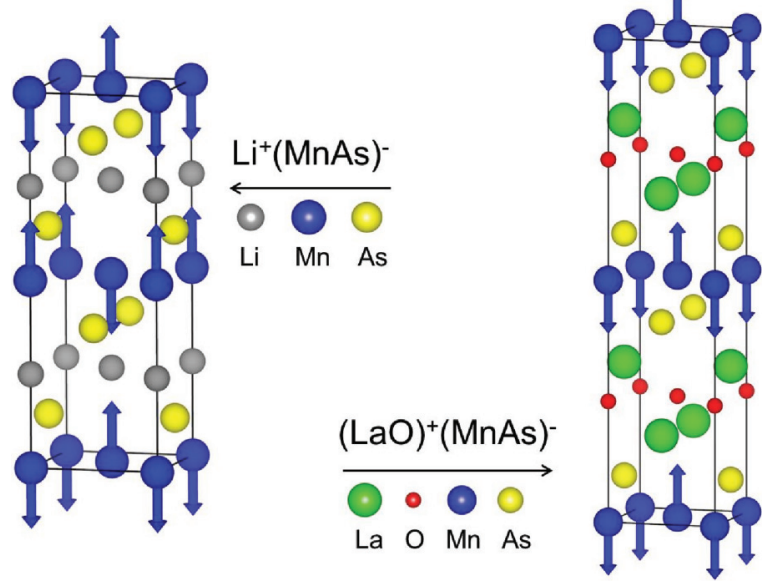

FIG. 1. (Color online) Crystal and magnetic structure of LiMnAs (Ref. 15) and LaOMnAs (Ref. 9). The blue arrows indicate the direction of the magnetic moments of the Mn atoms.

then stems from the nonbonding $d$ electrons, as can be best observed in half-Heusler compounds, where only Mn or rareearth-containing compounds form stable magnetic phases. ${ }^{18,19}$

The aim of this work is multifold: We elaborate on the synthesis and bulk characterization of polycrystalline LiMnAs and LaOMnAs, and compare their properties to other related compounds based on a short review from the literature on the $A \mathrm{Mn} X$ family. We show that LiMnAs can undergo a first-order phase transition from the tetragonal to a cubic phase. In addition, we discuss the electronic and magnetic properties of LiMnAs and LaOMnAs, including their transport properties. By combining ab initio density functional theory (DFT) calculations with our experiments, we discuss the electronic structure and magnetic exchange coupling of the $\mathrm{Mn}$ atoms, further commenting on the non-Curie-Weiss-like behavior experimentally reported for related compounds. ${ }^{20}$ We will also comment on the issue arising from a previous work $^{9}$ regarding the weak ferromagnetic behavior observed in LaOMnAs, which was first attributed to a small spin canting ${ }^{9}$ and in a later paper $^{21}$ explained via the formation of small amounts $(\leqslant 1 \%)$ of MnAs as impurities during synthesis.

\section{RESULTS AND DISCUSSION}

In Ref. 22 it was already noted that in the $A M n X$ series, where $A$ is an alkali metal and $X$ is an element from the 15 th group of the periodic table, there is a correlation between the local moments of $\mathrm{Mn}$ and the Mn-Mn distances $\left(d_{\mathrm{Mn}-\mathrm{Mn}}\right)$. That is, a shorter $d_{\mathrm{Mn}-\mathrm{Mn}}$ results in lower moment of Mn. The $d_{\mathrm{Mn}-\mathrm{Mn}}$ distance plays a major role in the magnetism of these compounds, whose behavior is transferable to LiMnAs, ${ }^{15}$ LaOMnAs, and other materials. The structural and magnetic data for the $A$ MnAs series derived from neutron diffraction measurements are collected from the literature in Table I.

Before turning to the presentation of our results, in Table II first we provide a general overview of the most important characteristics and material properties of various compounds for the aforementioned $A \mathrm{Mn} X$ class, with $A=\mathrm{Li}, \mathrm{LaO}$. Table II serves as a set of reference values, providing a solid basis of comparison with our measured data for LiMnAs
TABLE I. Short overview from the literature on the results obtained with neutron diffraction measurements on AMnAs compounds, where $A$ is an alkali metal.

\begin{tabular}{lccccc}
\hline \hline Compound & $T[\mathrm{~K}]$ & $a[\AA]$ & $c[\AA]$ & $d_{\mathrm{Mn}-\mathrm{Mn}}[\AA]$ & $m\left[\mu_{\mathrm{B}}\right]$ \\
\hline LiMnAs $^{\mathrm{a}}$ & 10 & $4.262(1)$ & $12.338(1)$ & $3.008(3)$ & $3.67(3)$ \\
NaMnAs $^{15}$ & 14 & $4.199(1)$ & $14.164(1)$ & $2.969(1)$ & $4.01(5)$ \\
KMnAs $^{15}$ & 14 & $4.382(1)$ & $15.558(1)$ & $3.099(1)$ & $4.03(5)$ \\
RbMnAs $^{20}$ & 10.6 & $4.400(5)$ & $16.128(5)$ & $3.111(1)$ & $4.05(4)$ \\
CsMnAs $^{22}$ & 10 & $4.417(1)$ & $17.370(1)$ & $3.123(1)$ & $4.07(2)$ \\
\hline
\end{tabular}

${ }^{\mathrm{a}}$ Results from this work.

and LaOMnAs, using which we can derive more general conclusions that are applicable to the entire family. As the data in Table II show, the materials in the $A \mathrm{Mn} X$ family can be classified into two main categories: those that are semiconducting or semimetallic with AFM ordering, and those which are metallic or half metallic and ferromagnetic (FM). There is a correlation between $d_{\mathrm{Mn}-\mathrm{Mn}}$ distances and the magnetic and electronic properties of these materials. In other words, those compounds that have $d_{\mathrm{Mn}-\mathrm{Mn}} \leqslant 3.1 \AA$ tend to be AFM semiconductors. In the range of $3.1 \leqslant d_{\mathrm{Mn}-\mathrm{Mn}} \leqslant 3.5 \AA$ they are semimetallic and AFM (except $\mathrm{CuMnSb}$ which has $d_{\mathrm{Mn}-\mathrm{Mn}}$ of $4.301 \AA$ ), and those with $d_{\mathrm{Mn}-\mathrm{Mn}} \geqslant 3.5$ are metallic and FM. As a general trend, this indicates that the further apart the $\mathrm{Mn}$ atoms are from each other, the more likely it is that they develop high moments, leading to a FM metal. Hence, by adjusting the lattice parameters, the magnetic and electronic properties of the $A \mathrm{Mn} X$ compounds can be tuned over a wide range. Indeed, as an example, for LaOMnP (isostructural to LaOFeAs), it was shown very recently that upon doping and external pressure, the system can undergo a phase transition from an AFM insulator to a metallic antiferromagnet. ${ }^{28}$

\section{A. Synthesis}

LiMnAs was synthesized and characterized as bulk material in the $1980 \mathrm{~s}^{15,29}$ by a high-temperature reaction of the elements lithium, manganese, and arsenic. It was found that this compound is AFM ordered, where the Néel temperature is approximately $400 \mathrm{~K}$, but the exact Néel temperature is not known.

Using a similar synthesis route, our preliminary experiments revealed a number of small Bragg reflections in powder $\mathrm{X}$-ray diffraction (XRD) patterns of the target compound caused by impurities. These Bragg peaks become significantly stronger with increasing temperature and reaction time and stem from an attack of the tantalum ampoule by arsenic. Therefore, LiMnAs was prepared as follows: Mixtures of stoichiometric amounts of $\mathrm{Li}$ (foil), Mn (chips), and As (pieces) (all 99.999\% purity) were placed in $\mathrm{Al}_{2} \mathrm{O}_{3}$ crucibles. The total mass was approximately $2 \mathrm{~g}$ per sample. Each crucible was enclosed in an arc-sealed tantalum ampoule at 300 mbar Ar, which in turn was jacketed by an evacuated fused silica ampoule. A gray powder was obtained after heating the mixtures to $1423 \mathrm{~K}$ with a low heating rate of $1 \mathrm{~K} / \mathrm{min}$ in a muffle furnace. This temperature was maintained for $2 \mathrm{~h}$ and then reduced to $1173 \mathrm{~K}$. After $24 \mathrm{~h}$, the ampoules were quenched in water. All of the handling was performed in a 
TABLE II. Structural, electronic, and magnetic properties of various $A \mathrm{Mn} X$-based compounds, where $A=\mathrm{Li}, \mathrm{Ni}, \mathrm{Cu}$, or $\mathrm{LaO}$ and $X=\mathrm{As}$ or $\mathrm{Sb}$, and the binary MnAs compound. The shortest Mn-Mn distance is given by $d_{\mathrm{Mn}-\mathrm{Mn}}$.

\begin{tabular}{|c|c|c|c|c|c|c|}
\hline Compound & $\begin{array}{l}\text { Electronic } \\
\text { structure }\end{array}$ & $\begin{array}{l}\text { Magnetic } \\
\text { order }\end{array}$ & $\begin{array}{l}\text { Mn magnetic } \\
\text { moment }\left[\mu_{\mathrm{B}}\right]\end{array}$ & $d_{\mathrm{Mn}-\mathrm{Mn}}[\AA]$ & $\begin{array}{l}\text { Space } \\
\text { group }\end{array}$ & Literature \\
\hline LiMnAs & semiconductor & AFM & 3.8 & 3.008 & $\begin{array}{c}P 4 / n m m \\
\text { (tetragonal) }\end{array}$ & this work and Ref. 15 \\
\hline CuMnAs & semimetal & AFM & no data & 3.452 & $\begin{array}{c}\text { Pnma } \\
\text { (orthorh.) }\end{array}$ & Ref. 12 \\
\hline $\mathrm{CuMnSb}$ & semimetal & AFM & 3.9 & 4.301 & $\begin{array}{l}F \overline{4} 3 m \\
\text { (cubic) }\end{array}$ & Refs. 12 and 23 \\
\hline LaOMnAs & semiconductor & AFM & 3.34 & 2.914 & $\begin{array}{c}P 4 / n m m \\
\text { (tetragonal) }\end{array}$ & Refs. 9 and 21 \\
\hline NiMnAs & $\begin{array}{l}\text { half metallic- } \\
\text { ferromagnet }\end{array}$ & FM & 4.0 & 4.051 & $\begin{array}{l}F \overline{43 m} \\
\text { (cubic) }\end{array}$ & Ref. 24 \\
\hline $\mathrm{NiMnSb}$ & $\begin{array}{l}\text { half metallic- } \\
\text { ferromagnet }\end{array}$ & FM & 4.0 & 4.186 & $\begin{array}{l}F \overline{4} 3 m \\
\text { (cubic) }\end{array}$ & Refs. 23 and 25 \\
\hline MnAs & metallic & FM & 3.4 & 3.730 & $\begin{array}{c}P 6_{3} / m m c \\
\text { (hexagonal) }\end{array}$ & Refs. 26 and 27 \\
\hline
\end{tabular}

glove box under $\operatorname{Ar}\left[p\left(\mathrm{O}_{2}, \mathrm{H}_{2} \mathrm{O}\right) \leqslant 1\right.$ ppm $]$. LaOMnAs was synthesized from the starting materials LaAs, $\mathrm{Mn}$, and $\mathrm{MnO}_{2}$ in stoichiometric amounts at $1423 \mathrm{~K}$ for $42 \mathrm{~h}$ in an $\mathrm{Al}_{2} \mathrm{O}_{3}$ crucible, which was in turn enclosed in an evacuated and fused silica tube.

\section{B. Crystal and magnetic structure}

Powder X-ray diffraction (XRD) of the final product showed single-phase LiMnAs. The powder diffraction experiment was performed with $\mathrm{Cu} \mathrm{K} \alpha_{1}$ radiation $(\lambda=1.5406 \AA)$ in Debye-Scherrer geometry. The sample was sealed in a glass capillary with a $0.3 \mathrm{~mm}$ diameter. A representative XRD pattern obtained at $293 \mathrm{~K}$ is shown in Fig. 2 with a Rietveld refinement carried out using Jana 2006. ${ }^{30}$ LiMnAs has a tetragonal unit cell with $a=4.2555(1) \AA$ and $c=6.1641(2) \AA$

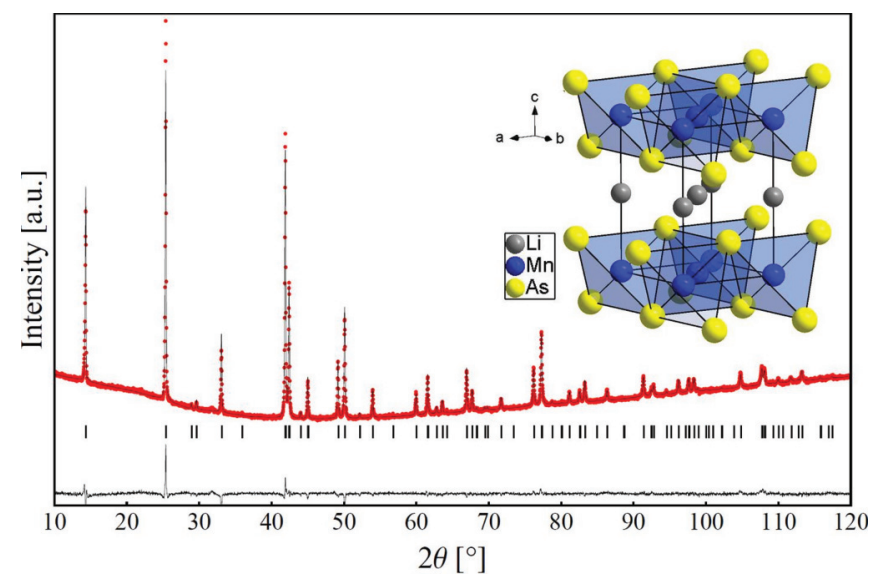

FIG. 2. (Color online) The observed x-ray powder diffraction pattern (points) and the fit from the Rietveld refinement (solid line) of LiMnAs at $293 \mathrm{~K}$. The markers of the Bragg reflections and the difference curve are shown at the bottom, respectively. The inset shows the tetragonal crystal structure highlighting slabs of edge-connected $\mathrm{MnAs}_{4}$ tetrahedra stacked along the $c$ axis.
$[P 4 / n m m, Z=2$, Li on $2 b$, Mn on $2 a$, As on $2 c$, and $z($ As $)=$ $0.7575(2)]$ in agreement with the older reports.

Chemical analysis for nonmetal impurities were carried out using the carrier gas hot extraction or the combustion technique [TCH 600, C 200 (LECO)]. For all samples, the impurities were below the limit of detection (LOD in ppm): $\mathrm{H}(100)$, $\mathrm{N}(200), \mathrm{O}(1000)$, and C(1500) based on $20 \mathrm{mg}$ initial weight. The 1:1:1 target composition was checked by inductively coupled plasma optical emission spectrometry (ICP-OES; Vista RL, Varian) resulting in Li:Mn:As = 1.03(1):0.98(1):0.99(1).

A specimen for metallographic examination was prepared from one sample. Because of moisture and air sensitivity, this was carried out in a glove box using paraffin oil as a lubricant. A bright field image of the microstructure is shown in Fig. 3(b), which reveals a domain structure. This is a typical signature for a tetragonal phase formed from a high-temperature cubic phase.

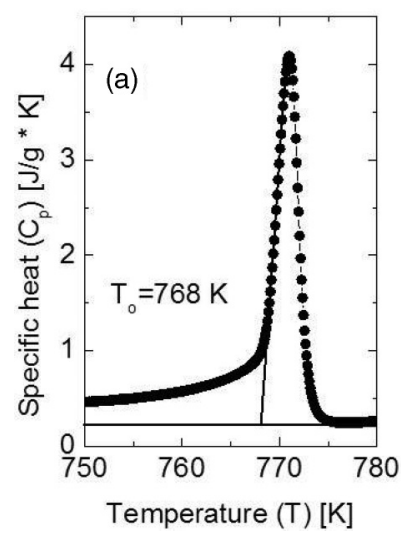

(b)

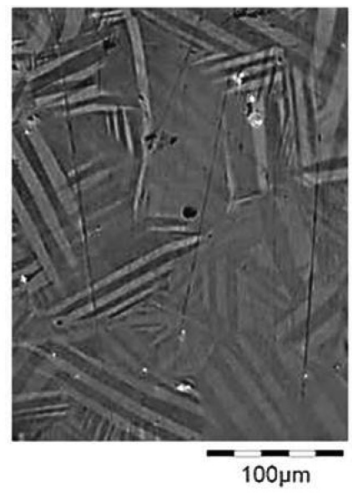

FIG. 3. (a) The DSC peak at $771 \mathrm{~K}$ with an onset temperature of $T_{\mathrm{o}}=768 \mathrm{~K}$ corresponds to the phase transformation to the cubic high-temperature phase. (b) Optical bright field image at room temperature showing the domain structure of LiMnAs induced by the phase transformation. 


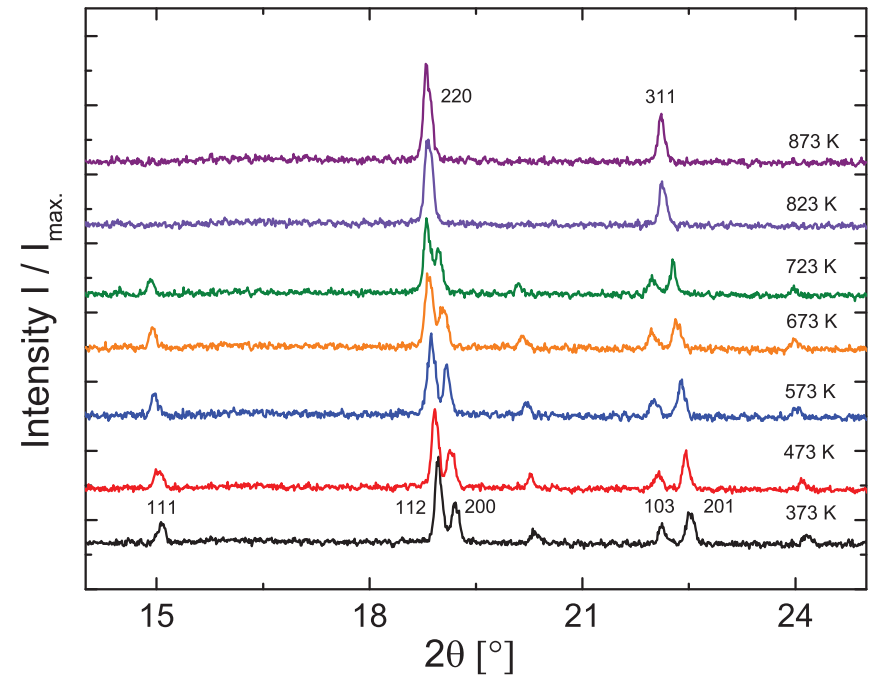

FIG. 4. (Color online) Observed x-ray powder patterns in the range $14^{\circ} \leqslant 2 \theta \leqslant 25^{\circ}$ of LiMnAs for various temperatures from $373 \mathrm{~K}$ to $873 \mathrm{~K}$. The patterns up to $723 \mathrm{~K}$ can be indexed primitive tetragonal. At $823 \mathrm{~K}$ and above, LiMnAs crystallizes in a face-centered-cubic structure.

Specific-heat measurements of LiMnAs in the temperature range of 240-780 K revealed only one DSC (differential scanning calorimetry) peak [see Fig. 3(a)] corresponding to a reversible phase transition with onset temperature $T_{\mathrm{o}}=768 \mathrm{~K}$. To identify the nature of this phase transition, temperaturedependent powder XRD measurements were performed with a Stoe STADI II diffractometer with Mo $\mathrm{K} \alpha_{1}$ radiation. The powder $(20-50 \mu \mathrm{m})$ was sealed in a $0.5 \mathrm{~mm}$ quartz glass capillary. The measurements were carried out in the range from $323 \mathrm{~K}$ to $873 \mathrm{~K}$ in steps of $50 \mathrm{~K}$. The observed powder patterns are shown in the range of $14^{\circ} \leqslant 2 \theta \leqslant 25^{\circ}$ in Fig. 4. All powder patterns up to $723 \mathrm{~K}$ can be indexed based on a tetragonal unit cell, whereas the powder patterns at $823 \mathrm{~K}$ and $873 \mathrm{~K}$ are indexed based on a cubic unit cell with $a_{\text {cub }} \approx c_{\text {tet }} \approx a_{\text {tet }} \sqrt{2}$. The high-temperature phase, HT-LiMnAs, crystallizes in MgAgAs structure type ( $F \overline{4} 3 m$, $Z=4, \mathrm{Li}$ on $4 b, \mathrm{Mn}$ on $4 a$, and As on $4 c$ ) according to the Rietveld analysis (see Fig. 5). The atomic positions are shown in Table III and the bond distances in Table IV, respectively.

The temperature dependence of the lattice parameters $a_{\text {cub }}, c_{\text {tet }}$, and $a_{\text {tet }} \sqrt{2}$ as well as the unit cell volume in a pseudocubic setting are plotted versus the temperature in Fig. 6. Both parameters $a$ and $c$ increase nearly linearly with $\alpha_{a \sqrt{2}}=1.57 \times 10^{-4} \pm 4 \times 10^{-6} \AA / \mathrm{K}$ and $\alpha_{c}=1.35 \times 10^{-3} \pm 4 \times 10^{-6} \AA / \mathrm{K}$ up to $600 \mathrm{~K}$. Above $650 \mathrm{~K}$, $a$ increases and $c$ decreases until they match at $768 \mathrm{~K}$, where $a \approx 6.101 \AA$. The volume increases linearly with the temperature expansion coefficient of $\alpha_{\mathrm{V}}=0.00832 \pm 1 \times 10^{-6} \AA^{3} / \mathrm{K}$.

The structural relationship between tetragonal LiMnAs and the high-temperature cubic form is shown in Fig. 7 with the tetragonal structure given in the pseudocubic setting. The crystal structure at room temperature form can be described as an alternate stacking of slabs composed of edge-connected slightly distorted $\mathrm{MnAs}_{4}$ and $\mathrm{LiAs}_{4}$ tetrahedra. The $\mathrm{MnAs}_{4}$ tetrahedra are slightly smaller than the $\mathrm{LiAs}_{4}$ tetrahedra. At higher temperatures the $c / a$ ratio in pseudocubic setting

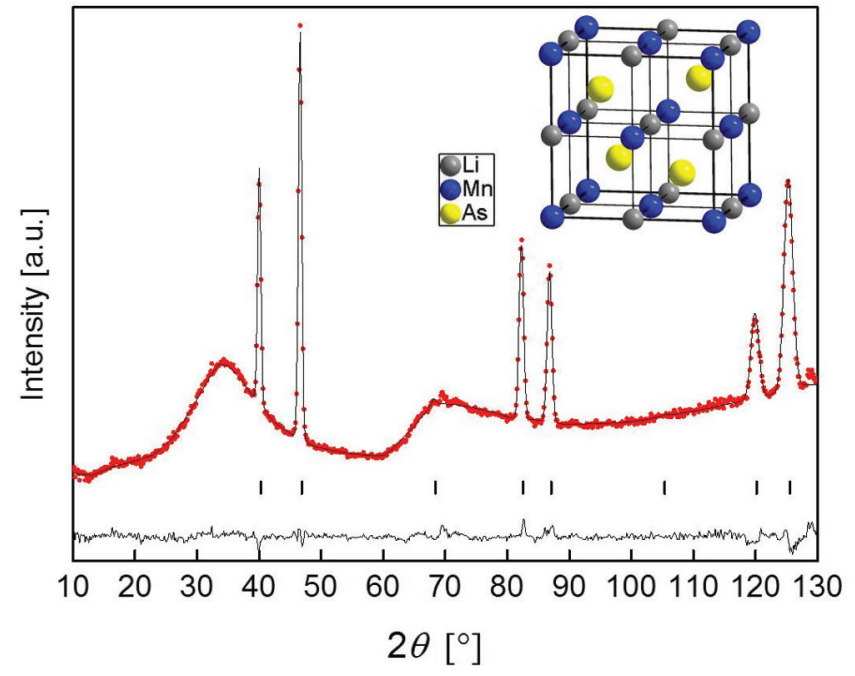

FIG. 5. (Color online) Observed neutron powder pattern (points) for LiMnAs at $873 \mathrm{~K}$ with the fit of the Rietveld refinement of the nuclear structure. The Bragg reflections marker and the difference curve are shown at the bottom. The inset shows the cubic crystal structure of LiMnAs. The best fitting exhibits an $R_{\text {profile }}$ of $2.1 \%, R_{\text {obs }}$ of $2.5 \%$, and the goodness of fit (GOF) of 1.3. The high background at low $2 \theta$ values is caused by the quartz glass ampule at high temperatures.

approaches one. At $768 \mathrm{~K}$, all tetrahedra become regular and half of the $\mathrm{Li}$ and $\mathrm{Mn}$ atoms of each layer exchange positions with each other in such a way that a half-Heusler structure is obtained. The diffusion of the $\mathrm{Li}$ atoms to the Mn sites and vice versa likely occurs via the empty $\mathrm{Li}_{4} \mathrm{As}_{4}$ cubes, showing that $\mathrm{Li}$ is rather mobile in LiMnAs. There is no perceptible discontinuity in the volume curve at the temperature of the phase transition, and $c / a$ transits smoothly to one. Although this fulfills the requirements for a second-order phase transition, it is unlikely that the fast exchange of $\mathrm{Li}$ and $\mathrm{Mn}$ is a continuous process, because $P 4 / \mathrm{nmm}$ is not a direct subgroup of $F \overline{4} 3 \mathrm{~m}$. This can therefore be only a first-order phase transition. The measured change in the entropy and the enthalpy at the phase transition temperature is $\Delta H_{\text {trans }}=2.19 \mathrm{~J} /(\mathrm{Kmol})$ $(\approx 23 \mathrm{meV} /$ f.u. $)$ and $\Delta S_{\text {trans }}=2.85 \mathrm{~J} /(\mathrm{Kmol})$.

For the magnetic characterization of LiMnAs, neutron powder diffraction patterns were recorded on the E6 focusing powder diffractometer at Helmholtz-Zentrum Berlin, Germany, using the wavelength $\lambda=2.448 \AA$. The measurements were performed at seven different temperatures in the range from $1.6 \mathrm{~K}$ to $873 \mathrm{~K}$. Five diffraction patterns in the range of $1.6 \mathrm{~K}$ to $300 \mathrm{~K}$ correspond to tetragonal

TABLE III. Fractional atomic coordinates and the anisotropic displacement parameters $(U)$ for the high-temperature cubic phase of LiMnAs.

\begin{tabular}{lccccc}
\hline \hline Atom & $\begin{array}{c}\text { Wyckoff } \\
\text { symbol }\end{array}$ & $x$ & $y$ & $z$ & $U\left[\AA^{2}\right]$ \\
\hline $\mathrm{Li}$ & $4 \mathrm{~b}$ & $\frac{1}{2}$ & $\frac{1}{2}$ & $\frac{1}{2}$ & $0.061(17)$ \\
$\mathrm{Mn}$ & $4 \mathrm{a}$ & 0 & 0 & 0 & $0.025(12)$ \\
$\mathrm{As}$ & $4 \mathrm{c}$ & $\frac{1}{4}$ & $\frac{1}{4}$ & $\frac{1}{4}$ & $0.021(4)$ \\
\hline \hline
\end{tabular}


TABLE IV. Selected bond distances for the cubic phase of LiMnAs at $873 \mathrm{~K}$.

\begin{tabular}{lc}
\hline \hline Atom-Atom & Distance $[\AA]$ \\
\hline Li-As & $2.665(1)$ \\
Mn-Li & $3.077(2)$ \\
As-Mn & $2.665(1)$ \\
\hline \hline
\end{tabular}

and antiferromagnetically ordered LiMnAs. To protect the sample against moisture and oxidation, a $10 \mathrm{~mm}$ fused silica tube was filled and sealed under vacuum. A representative neutron powder diffraction pattern obtained at $1.6 \mathrm{~K}$ is shown in Fig. 8 along with the fit from the Rietveld refinement using the nuclear and magnetic structures. Table $\mathrm{V}$ lists crystallographic data obtained from the Rietveld refinements of the crystal structures based on neutron powder patterns at various temperatures. The powder patterns below the ordering temperature reveal Bragg peaks of magnetic order that can be indexed with the modulation vector $\vec{k}=\left(0,0, \frac{1}{2}\right)$. This corresponds to an antiferromagnetically ordered structure doubling the $c$ axis of the nuclear structure. The refinement in the magnetic space group $P_{C} 4_{2} / \mathrm{ncm}$ (Belov notation) reveals that the magnetic moments at the Mn sites with $3.7 \mu_{\mathrm{B}}$ at $1.6 \mathrm{~K}$ are aligned parallel to the $c$ axis. These observations are in good agreement with the previously reported data, except that the magnetic space group was wrongly assigned in Ref. 15 . The low-temperature magnetic moment of $3.7 \mu_{\mathrm{B}}$ is smaller

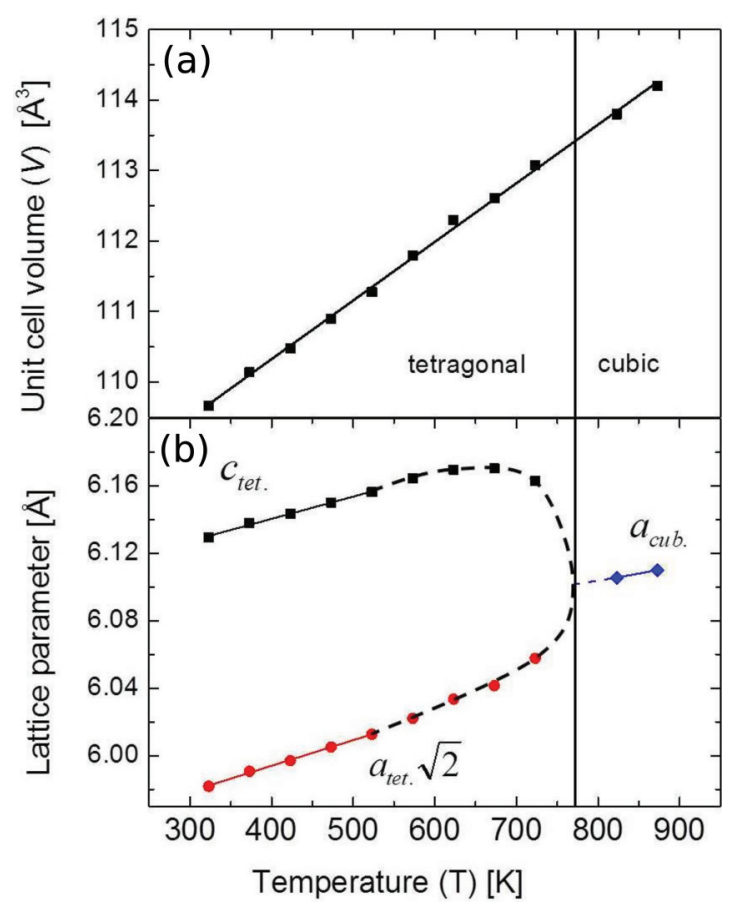

FIG. 6. (Color online) (a) The unit cell volume of LiMnAs $(Z=4)$ increases linearly from $373 \mathrm{~K}$ to $873 \mathrm{~K}$. There is no perceptible volume jump at the phase transition temperature. (b) Lattice parameter (from the XRD measurements on LiMnAs) versus temperature for the tetragonal and cubic phases. Below $600 \mathrm{~K}$, the curves are fitted by a linear function. The dashed line at a higher temperature serves as a guide to the eye.

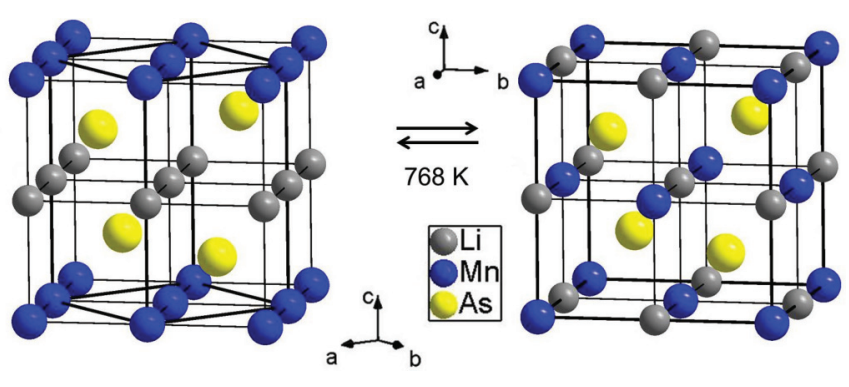

FIG. 7. (Color online) Crystal structures of LiMnAs: (a) tetragonal primitive and (b) face centered.

than the value of $5 \mu_{\mathrm{B}}$ expected for the high-spin $(S=5 / 2)$ state of $\mathrm{Mn}^{2+}$, assuming a $g$ factor of $2\left(\mu=g S \mu_{\mathrm{B}}\right)$.

Figure 9 shows the temperature dependence of the magnetic moment $m$ obtained from the refinement of the neutron diffraction data. All data below $380 \mathrm{~K}$ were fitted by the power law of $m(T)=m_{0}\left(1-T / T_{N}\right)^{\beta}$ with Néel temperature $T_{N}$ $=373.77 \mathrm{~K}$ and the critical exponent $\beta=0.239$. Regarding the magnetism of LaOMnAs, in Ref. 9, it was found that it is antiferromagnetically ordered in the $a b$ plane, similar to LiMnAs, according to neutron diffraction analysis at lower temperatures. The small increase in the magnetic moments was explained ${ }^{9}$ by a weak ferromagnetic behavior as a result of a small spin canting. Such canting could be caused by broken inversion symmetry, giving rise to the DzyaloshinskiiMoriya $^{31,32}$ interaction modifying the magnetic ground state, which ceases to remain collinear. However, in LiMnAs and $\mathrm{LaOMnAs}$, the inversion symmetry break occurs anyway at their surfaces or interfaces. Thus, in bulk LaOMnAs we would expect that canting plays a negligible role. Instead of canting, in agreement with Ref. 21 we explain the small remanent total moment of these compounds with the formation of MnAs as an impurity phase. This is because during synthesis, independent of the method used, tiny amounts of MnAs $(\leqslant 1 \%)$ can easily be formed, which cannot be detected by techniques such as XRD or energy-dispersive x-ray spectroscopy (EDX). As shown by

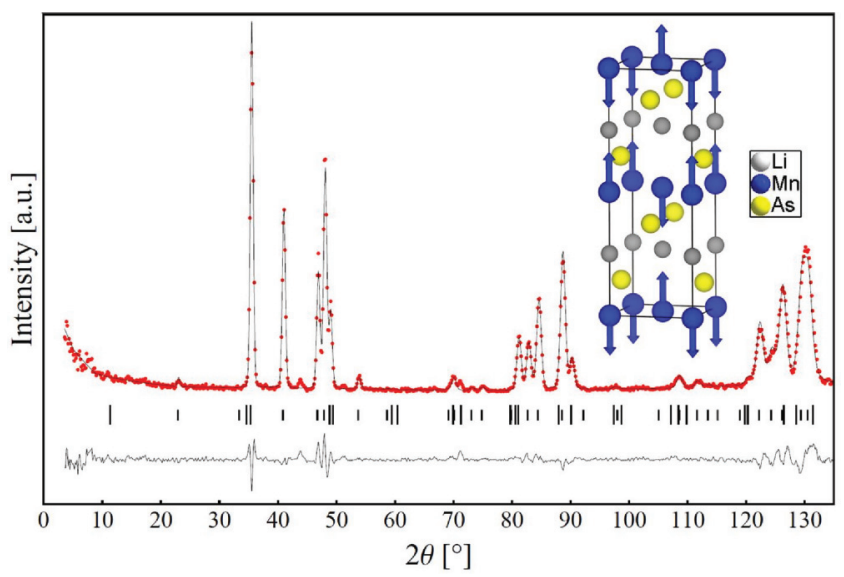

FIG. 8. (Color online) Observed neutron powder pattern (points) for LiMnAs at $1.6 \mathrm{~K}$ with the fit of the Rietveld refinement of the nuclear and magnetic structure. The markers of the Bragg reflections for the nuclear reflections (short lines), magnetic reflections (long lines), along with the difference curve are shown at the bottom. The inset shows the alignment of the magnetic moments along the $c$ axis. 
TABLE V. Crystallographic data for tetragonal antiferromagnetically ordered and tetragonal paramagnetic LiMnAs and for the high-temperature cubic phase of LiMnAs. The $z$ parameter for As in the antiferromagnetically ordered structure is half of that of the paramagnetic structure. In a cubic setup it is defined as $1-z=\frac{1}{4}$. For comparison, the magnetic moment of $\mathrm{Mn}^{2+}$ determined from neutron diffraction measurements is also given together with data from Ref. 15. The residual factors, $R_{\mathrm{B}}$, for the magnetic and nuclear parts are not provided because they are nearly equal for each measurement (approximately 6\%).

\begin{tabular}{lccccc}
\hline \hline$T[\mathrm{~K}]$ & $a[\AA]$ & $c[\AA]$ & $V\left[\AA^{3}\right]$ & $z$ & $m\left[\mu_{\mathrm{B}}\right]$ \\
\hline 1.6 & $4.262(1)$ & $12.338(1)$ & 224.12 & $0.3787(5)$ & $3.72(3)$ \\
10 & $4.262(1)$ & $12.338(1)$ & 224.12 & $0.3795(5)$ & $3.68(3)$ \\
$13^{15}$ & $4.253(1)$ & $12.310(1)$ & 222.66 & $0.3800(1)$ & $3.75(3)$ \\
30 & $4.262(1)$ & $12.338(1)$ & 224.12 & $0.3796(5)$ & $3.70(3)$ \\
50 & $4.261(5)$ & $12.337(9)$ & 224.00 & $0.3789(1)$ & $3.61(3)$ \\
$293^{15}$ & $4.267(1)$ & $12.356(1)$ & 224.97 & $0.3800(1)$ & $2.59(2)$ \\
300 & $4.259(2)$ & $12.333(4)$ & 223.70 & $0.3813(1)$ & $2.63(3)$ \\
317 & $4.269(2)$ & $12.363(4)$ & 225.20 & $0.3792(1)$ & $2.40(3)$ \\
322 & $4.269(2)$ & $12.366(4)$ & 225.40 & $0.3796(1)$ & $2.41(3)$ \\
332 & $4.270(2)$ & $12.372(4)$ & 225.60 & $0.3778(1)$ & $2.25(3)$ \\
348 & $4.272(2)$ & $12.374(4)$ & 225.80 & $0.3771(1)$ & $2.05(3)$ \\
352 & $4.273(2)$ & $12.373(4)$ & 225.80 & $0.3800(1)$ & $1.90(3)$ \\
357 & $4.274(2)$ & $12.377(4)$ & 226.00 & $0.3783(1)$ & $1.74(3)$ \\
362 & $4.273(2)$ & $12.380(4)$ & 226.00 & $0.3799(1)$ & $1.61(3)$ \\
366 & $4.276(2)$ & $12.380(3)$ & 226.40 & $0.3802(4)$ & $1.49(3)$ \\
370 & $4.275(3)$ & $12.383(3)$ & 226.20 & $0.3783(4)$ & $1.15(3)$ \\
373 & $4.275(2)$ & $12.382(3)$ & 226.20 & $0.3814(3)$ & $0.91(3)$ \\
$393^{\mathrm{a} 15}$ & $4.273(1)$ & $12.370(1)$ & 225.86 & $0.3800(1)$ & $1.59(3)$ \\
$423^{15}$ & $4.279(1)$ & $6.193(1)$ & 113.39 & $0.7610(2)$ & \\
573 & $4.289(5)$ & $6.203(0)$ & 114.11 & $0.7582(1)$ & \\
873 & $6.155(4)$ & & 233.23 & $\frac{1}{4}$ & \\
\hline \hline
\end{tabular}

${ }^{a}$ Magnetic moment not compatible with our data.

the data in Table II, MnAs is an ordered FM material. It has a Curie temperature of approximately $320 \mathrm{~K},{ }^{26}$ exactly coinciding with the magnetic transition temperature in our measurements.

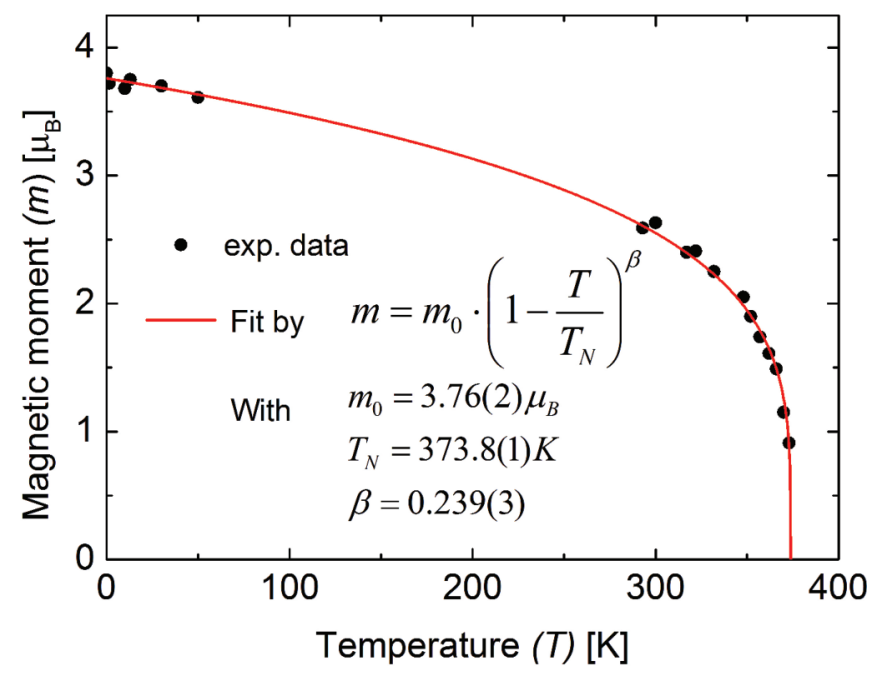

FIG. 9. (Color online) Ordered Mn magnetic moment $m$ of LiMnAs as a function of temperature $T$. The solid curve through the data is fitted to a power-law function with critical exponent $\beta$.
Thus, the presence of MnAs impurities in the samples offers a more straightforward explanation for the small remanent total moments reported in Ref. 9, rather than spin canting.

Regarding the non-Curie-Weiss-like behavior of the magnetization as a function of temperature reported for related $A \mathrm{Mn} X$ compounds ${ }^{20}$ our DFT calculations (see below) have shown that within the layers, the Mn moments are very strongly coupled relative to the weak interlayer couplings. Additionally, in LiMnAs between the layers, the Mn atoms are actually coupled ferromagnetically with their next-nearest neighbors and antiferromagnetically with their second-nearest neighbors. Because of this complex interaction between Mn moments, even above the Néel temperature, a short-range local order is present, causing the non-Curie-Weiss-like behavior described above. In comparison with theory, DFT calculations (see below) yield a value of $3.8 \mu_{\mathrm{B}}$ for the antiferromagnetically ordered structure at $0 \mathrm{~K}$. In the refinement, the magnetic form factor for $\mathrm{Mn}^{2+}$ has been used. Instead of $\mathrm{Mn}^{2+}$, taking the magnetic form factor for $\mathrm{Mn}^{+}$results in a slight correlation with the magnetic moments, where the magnetic moment of $\mathrm{Mn}$ is enhanced by about $0.1-0.2 \mu_{\mathrm{B}}$. However, in LiMnAs, referring to $\mathrm{Mn}^{2+}$ is chemically more logical. This is supported by analyzing the $\mathrm{x}$-ray absorption nearedge structure spectroscopy (XANES) data of several Mn compounds including LiMnAs.

The environment and the valence state of the Mn atoms in LiMnAs have been investigated by means of extended $\mathrm{x}$-ray absorption fine-structure (EXAFS) and XANES at the $\mathrm{Mn}$ edge $(6539 \mathrm{eV}) \mathrm{K}$ at room temperature in the transmission arrangement at the EXAFS beamline A1 of the Hamburg synchrotron radiation laboratory, HASYLAB, at DESY. Wavelength selection was realized by means of a $\mathrm{Si}(111)$ double-crystal monochromator. The near-edge region of $6510-6560 \mathrm{eV}$ was measured with a minimal step size of $0.25 \mathrm{eV}$, and the EXAFS regime was measured up to $7540 \mathrm{eV}$. A mass of approximately $10 \mathrm{mg}$ of powdered Mn compounds with a particle size of $\leqslant 20 \mu \mathrm{m}$ was diluted with $\mathrm{B}_{4} \mathrm{C}$ powder and mixed with polyethylene powder. The mixture was pressed to form a pellet with a diameter of $10 \mathrm{~mm}$. All of the handling was performed under an argon atmosphere in a glove box. Reference data were measured simultaneously with a Mn foil. This spectrum serves as an external reference for energy calibration and Mn valency. Figure 10 shows the EXAFS signal, $\chi(k)$, and the corresponding Fourier transform. The EXAFS data were analyzed in accordance with the standard procedure for data reduction, ${ }^{33}$ using IFFEFIT. ${ }^{34}$ The FEFF program was used to obtain the phase shift and amplitudes. ${ }^{35}$ The EXAFS signal $\chi(k)$ was extracted and Fourier transformed (FT) using a Kaiser-Bessel window with a $\Delta(k)$ range of $7.0 \AA^{-1}$. The $\chi(k)$ curve shows the characteristic pattern for this compound. The FT pattern shows one broad peak at approximately $2.2 \AA$ (uncorrected for the phase shift), which corresponds to the Mn-As and Mn-Mn scattering contributions. The Mn-Li scattering contribution is also included in this region; however, it does not have a strong influence on the main peak. The gray line in Fig. 10 represents the best-fitting curve of the data. The structural parameters obtained from the Rietveld refinement were used as the input data to generate the cluster of atoms used. Only single-scattering events were considered in the fitting procedure. The excellent agreement between the data 

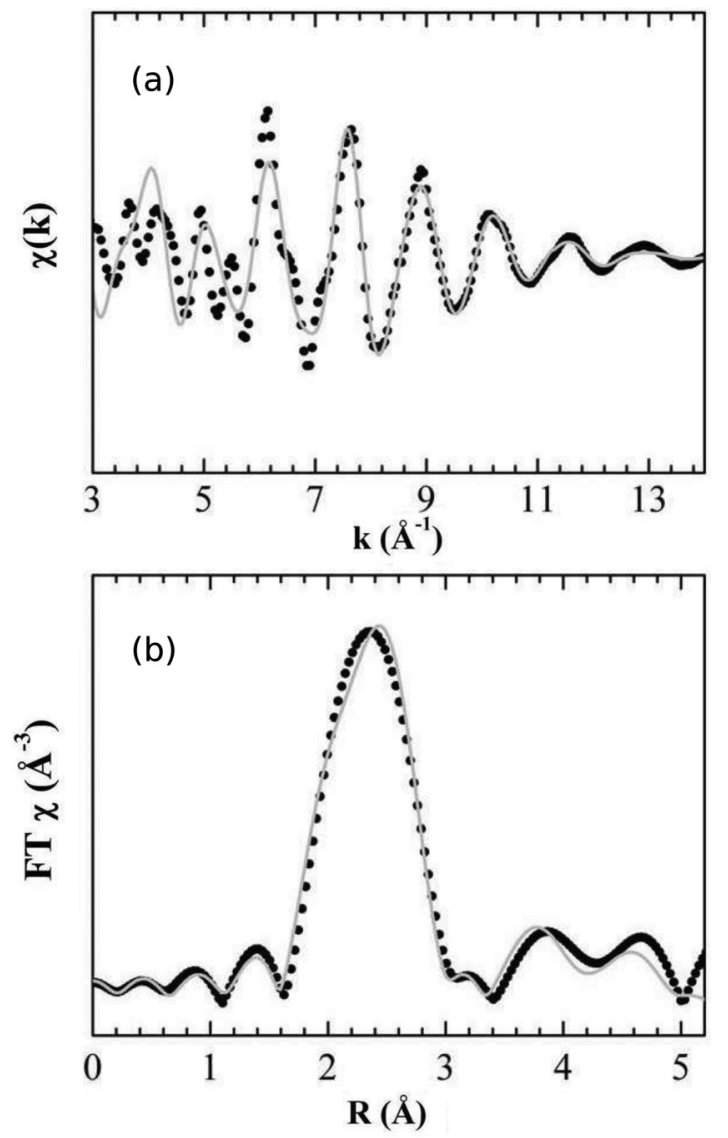

FIG. 10. (a) $293 \mathrm{~K}$ Mn EXAFS (points) and the corresponding best fit (solid line) of LiMnAs. (b) Fourier transform of the $293 \mathrm{~K}$ Mn EXAFS (points) and the corresponding best fit (solid line) of LiMnAs.

and the theoretical structure was accomplished by the low $R$ factors $(\leqslant 0.6 \%)$. The deviation between data and theory in the $\chi(k)$ at low $k\left(6 \AA^{-1}\right)$ is due to the short-wavelength components corresponding to higher coordination shells.

A quantitative analysis extracted from the EXAFS data is summarized in Table VI. The amplitude-reduction term $\left(S_{0}\right)^{2}$ used was 0.75 . The Mn-As and Mn-Mn distances are slightly contracted by $\leqslant 2 \%$ and the Mn-Li by $\approx 7 \%$ compared to the crystallographic data.

In Table VI, the distances obtained by the neutrondiffraction refinement are compared with the EXAFS data. The oxidation state of $\mathrm{Mn}$ in LiMnAs was obtained by using the XANES analysis with a widely used method as described

TABLE VI. Quantitative results for LiMnAs from the EXAFS data analysis for the Mn $k$ edge, considering the coordination numbers $(C N)$ of the crystal structure. The best fitting exhibits the next-neighbor distances $(R)$, mean-square displacement in $R \sigma^{2}$, and $R$ factor for the whole fit.

\begin{tabular}{lcccccc}
\hline \hline Shell & Element & $C N$ & $d[\AA]$ & $R_{\text {EXAFS }[\AA]}$ & $\sigma^{2}\left[\AA^{2}\right]$ & $R[\%]$ \\
\hline 1st & As & 4 & 2.601 & 2.550 & 0.006 & \\
2nd & $\mathrm{Mn}$ & 4 & 3.012 & 2.953 & 0.006 & 0.6 \\
3rd & $\mathrm{Li}$ & 2 & 3.085 & 2.870 & 0.006 & \\
\hline \hline
\end{tabular}

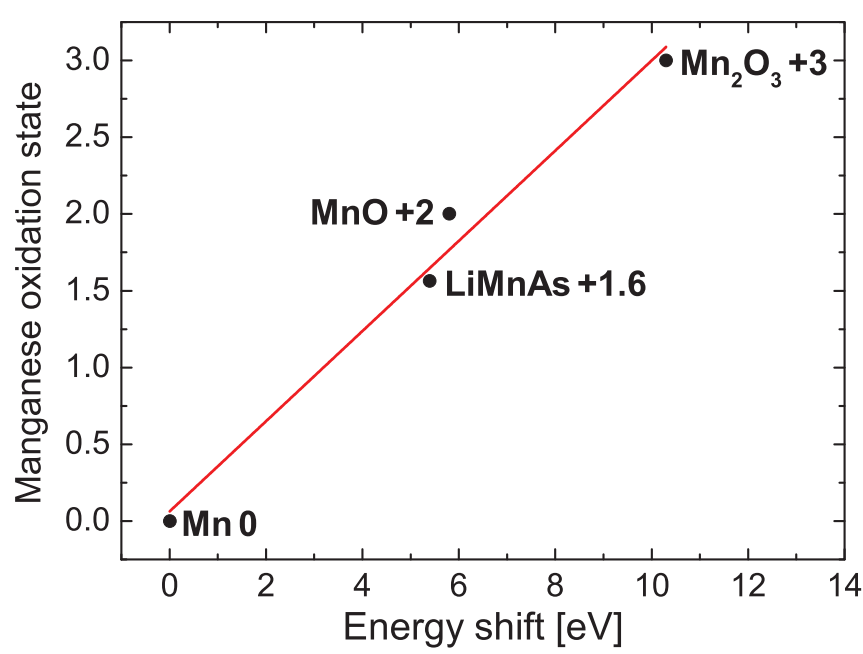

FIG. 11. (Color online) Plot of the oxidation state of $\mathrm{Mn}$ in elemental manganese and in various compounds versus the energy shift obtained from XANES measurements.

by Wong et al. ${ }^{36}$ This method considers the determination of the edge shift in relation to the $\mathrm{Mn}^{0}$ edge energy for a given compound. In this case, there is a linear relation between this energy shift and the oxidation state. Using the oxidation state and energy shift of some standard oxides $\left(\mathrm{Mn}_{2} \mathrm{O}_{3}\right.$ and $\mathrm{MnO}$ in this case), it is possible to find a linear relation and obtain the oxidation state for the compound (Fig. 11). This method reveals an oxidation state of Mn in LiMnAs of 1.6. Such a decrease in the oxidation state can be expected from weak homonuclear Mn-Mn interactions in the $a b$ plane. This is supported by DFT calculations (see below), which show a considerable interaction in the planes of $\mathrm{Mn}$ atoms, affecting their hybridization.

\section{C. dc resistivity}

The resistivity of polycrystalline LiMnAs powder was recorded using the "van der Pauw" method in the temperature range of 50-250 K, above which the LiMnAs sample has decomposed. For LaOMnAs, the electrical resistivities $\rho(T)$ from $100-870 \mathrm{~K}$ were obtained by a standard linear four-point contact method by using $2 \times 2 \times 8 \mathrm{~mm}^{3}$ bars of LaOMnAs cut from pellets obtained by a spark plasma sintering (SPS) process. The measurement was performed by means of a physical property measurements system (PPMS; Quantum Design model 6000, supported by LOT Germany).

Both LiMnAs and LaOMnAs show very high resistivity below about $100 \mathrm{~K}$. The resistivity measurements suggest a semiconducting behavior. The Arrhenius equation and an Arrhenius plot were used in order to obtain the activation energy, which has a value of approximately half of the band gap energy $E_{g}$ for intrinsic semiconductors ${ }^{37}$ according to the relation

$$
\sigma_{\mathrm{dc}} \propto \exp \left(-\frac{E_{\mathrm{g}}}{2 k_{\mathrm{B}} T}\right),
$$

where $k_{\mathrm{B}}=$ Boltzmann constant $\left(8.617332 \times 10^{-5} \mathrm{eV} / \mathrm{K}\right), T$ is the absolute temperature, and $E_{\mathrm{g}}$ is the band gap width. Figure 12 shows the Arrhenius plot and the temperature 


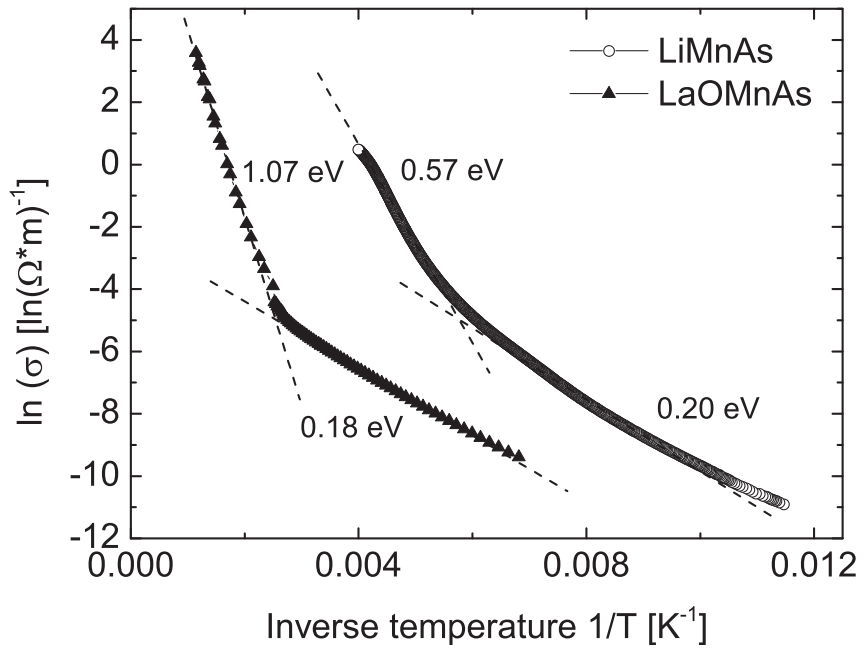

FIG. 12. Arrhenius plot of the electrical conductivity for LiMnAs and LaOMnAs. At low temperatures, the curves correspond to the extrinsic regime, and at high temperature, (low $1 / T)$, to the intrinsic regime.

dependence of the conductivity measurements for LiMnAs and LaOMnAs. In the resistivity measurements both samples show the same behavior, namely, two distinct sections of activated conduction with a small $E_{\mathrm{g}}$ of $0.20 \mathrm{eV}$ for LiMnAs and an $E_{\mathrm{g}}$ of $0.18 \mathrm{eV}$ for LaOMnAs, which indicate doped levels at lower temperatures and a larger $E_{\mathrm{g}}$ of $0.57 \mathrm{eV}$ for LiMnAs and an $E_{\mathrm{g}}$ of $1.07 \mathrm{eV}$ for LaOMnAs respectively, at higher temperatures.

Figure 13 shows the resistivity as a function of temperature on a logarithmic scale. We note in particular that the electrical resistivity changes by more than five orders of magnitude for LiMnAs in the temperature range of 100$250 \mathrm{~K}$ and by about two orders of magnitude for LaOMnAs from $150-350 \mathrm{~K}$.

\section{Dielectric permittivity}

Ellipsometric measurements covering the far-infrared to deep-ultraviolet spectral range $(10 \mathrm{meV}-6.5 \mathrm{eV})$ were carried

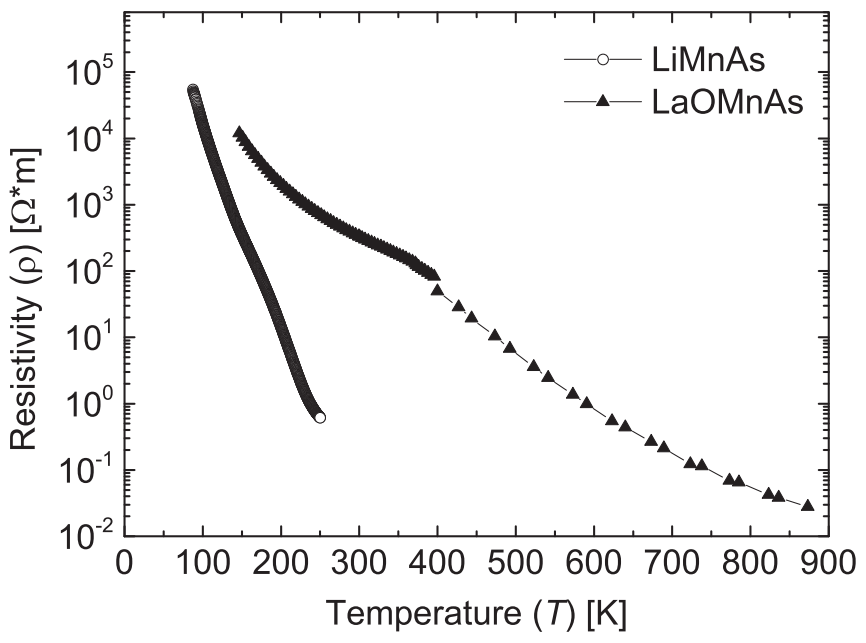

FIG. 13. Temperature dependence of the electrical resistivity, $\rho(T)$, on a logarithmic scale for LiMnAs and LaOMnAs.

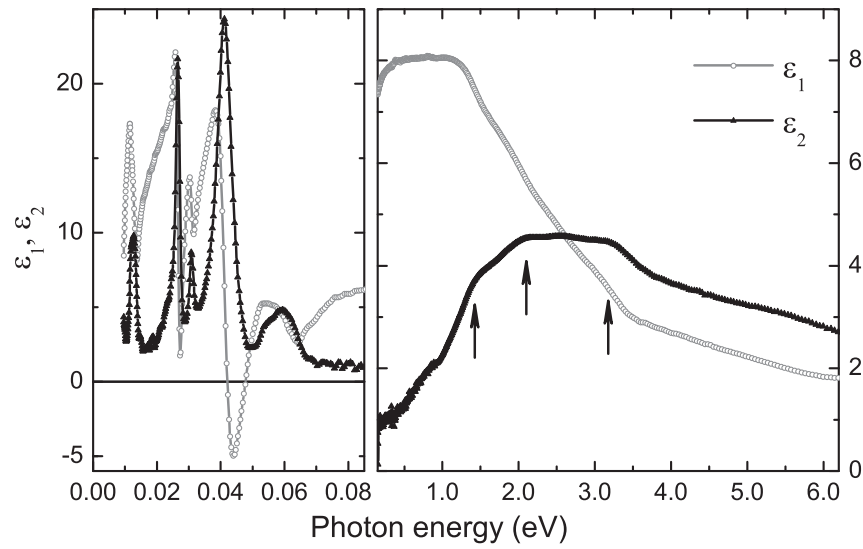

FIG. 14. (Left) Phonon and (right) interband electronic contribution to the real and imaginary parts of the dielectric function $\tilde{\varepsilon}(\omega)=\varepsilon_{1}(\omega)+i \varepsilon_{2}(\omega)$ of LaOMnAs measured at $T=294 \mathrm{~K}$. Arrows mark the peak positions of the main absorption bands.

out at room temperature on LaOMnAs ceramic dense pellets polished to an optical grade finish. The measurements in the near-infrared to deep ultraviolet spectral range $(0.75-6.5 \mathrm{eV})$ were performed with a rotating analyzer-type Woolam VASE variable-angle ellipsometer. For the infrared measurements from $0.01 \mathrm{eV}$ to $1.0 \mathrm{eV}$, we used home-built ellipsometers in combination with a Bruker Vertex 80v FT-IR spectrometer. Some of the experiments were performed at the infrared IR-1 beamline of the ANKA synchrotron light source at the Karlsruhe Institute of Technology, Germany. The complex dielectric function, $\tilde{\varepsilon}(\omega)=\varepsilon_{1}(\omega)+i \varepsilon_{2}(\omega)$, was directly determined from ellipsometric angles $\Psi(\omega)$ and $\Delta(\omega){ }^{38}$ The inversion of the ellipsometric data was performed within the framework of an effective medium approximation, which in the case of polycrystalline samples corresponds to the volume average of the anisotropic dielectric tensor projections. We did not take into account the surface roughness grain texturing effects on $\tilde{\varepsilon}(\omega)$, which is estimated to be less than $10 \%$ over the measured spectral range. Because of the air sensitivity, it was not possible to obtain ceramics of LiMnAs sufficiently dense for optical measurements.

Figure 14 shows the complex dielectric function of the LaOMnAs sample at $T=294 \mathrm{~K}$. In the far-infrared range, there are six strong phonon modes. The lowest interband transition in this material lies above $0.4 \mathrm{eV}$. One can clearly distinguish three optical bands peaked at $\sim 1.4, \sim 2.1$, and $\sim 3.2 \mathrm{eV} .{ }^{39}$ These transitions provide the main contribution to the static permittivity and form the direct absorption edge, below which the real part of the dielectric permittivity becomes nearly constant down to the phonon frequencies, $\varepsilon_{1}(0.2 \mathrm{eV} \lesssim \omega \lesssim 1.2 \mathrm{eV}) \approx 8$. We note that the main features resemble those that characterize the interband electronic contribution to the dielectric function of polycrystalline $\mathrm{LaOFeAs}$, while shifted by $\sim 0.7 \mathrm{eV}$ to higher energy. ${ }^{39}$ The rise of $\varepsilon_{2}(\omega)$ above $0.4 \mathrm{eV}$ can be attributed to indirect interband transitions.

\section{E. DFT calculations}

In order to gain further insights into the electronic structure and energetics of LiMnAs and LaOMnAs, we have performed 
$a b$ initio calculations based on the density functional theory (DFT) with the VASP ${ }^{40,41}$ code. For the approximate treatment of the electron exchange and correlation, we have used the Perdew, Burke, Ernzerhof (PBE) functional ${ }^{42}$ together with the projector-augmented wave (PAW) method with a plane-wave cutoff energy of $400 \mathrm{eV}$. In the irreducible part of the Brillouin zone, the $k$-point integration was performed on an $8 \times 8 \times 4$ Monkhorst-Pack mesh. To describe the magnetic ordering in the compounds as found from neutron diffraction for the tetragonal antiferromagnetic structures, spin-polarized calculations have been carried out for supercells containing four primitive unit cells (see Fig. 1).

In the case of LiMnAs, the calculated local Mn moments of $3.82 \mu_{\mathrm{B}}$ for the ground state are in excellent agreement with the value of $3.72 \mu_{\mathrm{B}}$ measured at $1.6 \mathrm{~K}$, which is the most representative temperature for a comparison between experiment and theory. As already mentioned in the introduction, the magnetic moment of the Mn atoms is well localized in this class of materials. Based on a fully ionic picture of LiMnAs, i.e., $\mathrm{Li}^{+}, \mathrm{Mn}^{2+}, \mathrm{As}^{3-}$, one would expect that the oxidation state of $\mathrm{Mn}$ to be $2^{+}$. The experimentally obtained value of $1.6^{+}$from XANES measurements is somewhat lower than this $2^{+}$expected value. Therefore, we have looked into the real-space density of LiMnAs, and apart from the expected $s p^{3}$ hybridization between $\mathrm{Mn}$ and As (see left panel in Fig. 15), we found that there is also a feature present that corresponds to a mixture between $\mathrm{Mn}-\mathrm{Mn}$ bonds and $\mathrm{Mn}$-As bonds (see right panel in Fig. 15).

From subsequent DFT calculations, we have determined the magnetic exchange coupling constants $J_{i j}$ of the classical Heisenberg model, $H=-\sum_{i>j} J_{i j} \hat{\mathrm{e}}_{i} \hat{\mathrm{e}}_{j}$, where $\hat{\mathrm{e}}_{i, j}$ are the unit vectors corresponding to the directions of the local magnetization at sites $i$ and $j$. For this purpose, we have employed the SPR-KKR program package, ${ }^{43}$ which is a suitable numerical tool for such computational task, not relying on pseudopotentials, where $J_{i j}$ can be evaluated within the Green's function formalism. (a)

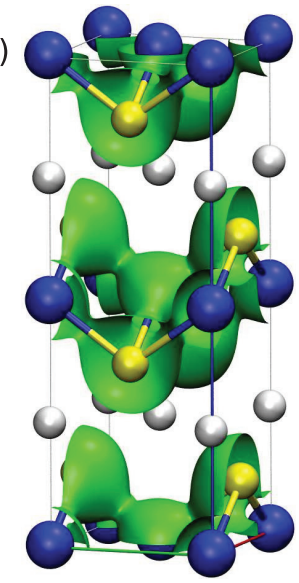

(b)

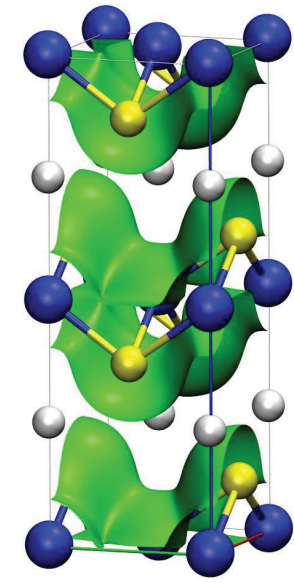

FIG. 15. (Color online) Real-space electronic density of LiMnAs. The density as green surfaces superimposed on top of the atomic structure at isovalues of $5.96 \times 10^{-3}$ and $3.58 \times 10^{-3} e / \AA^{3}$, corresponding (a) to $s p^{3}$ hybridization and (b) to a mixture between Mn-As and Mn-Mn bonding.
As follows from analysis of the exchange coupling scheme (see Fig. 16), within each Mn plane, the magnetic moments of the nearest neighbors are coupled antiparallel (with $J_{1} \approx-60 \mathrm{meV}$, shown in red), and this strong antiparallel coupling cannot be perturbed by the competing but smaller next-nearest neighbor antiparallel interaction $\left(J_{2} \approx-14.7\right.$ $\mathrm{meV}$, shown in dark red). Thus, within each plane the nearest Mn magnetic moments are ordered antiferromagnetically. Further, to understand how the nearest planes are magnetically oriented to one another, we consider the interplane exchange coupling. Both leading constants of the interplane exchange are positive, $j_{1,2} \approx 0.6$ and $0.24 \mathrm{meV}$, which correspond to the nearest and next-nearest interplane interactions, respectively. It is easy to see that for an already fixed in-plane magnetic order, these two interactions are also competing. Despite the fact that $j_{1}>j_{2}$, each $\mathrm{Mn}$ atom (for simplicity we consider one in the center of Fig. 16) has only 2 nearest neighbors from the two adjacent Mn planes (see $j_{1}$ represented as light green lines in Fig. 16), but there are 8 next-nearest interplane neighbors (see $j_{2}$ shown with dark green), which leads to an overall domination of the next-nearest interplane coupling $\left(8 j_{2}>2 j_{1}\right)$; thus, the next-nearest $\mathrm{Mn}$ planes are coupled antiferromagnetically.

At the same time, calculations for LaOMnAs system show that whereas the nearest in-plane $\mathrm{Mn}$ neighbors are coupled antiparallel (similar to LiMnAs case), the adjacent planes prefer to couple parallel to one another. The mechanism of this coupling is the following: similar to LiMnAs, two nearest interplane neighbors couple parallel to the central $\mathrm{Mn}$ atom $\left(j_{1}=+0.4 \mathrm{meV}\right)$, whereas 8 next-nearest neighbors, in contrast to LiMnAs, antiparallel $\left(j_{2}=-0.23 \mathrm{meV}\right)$, which

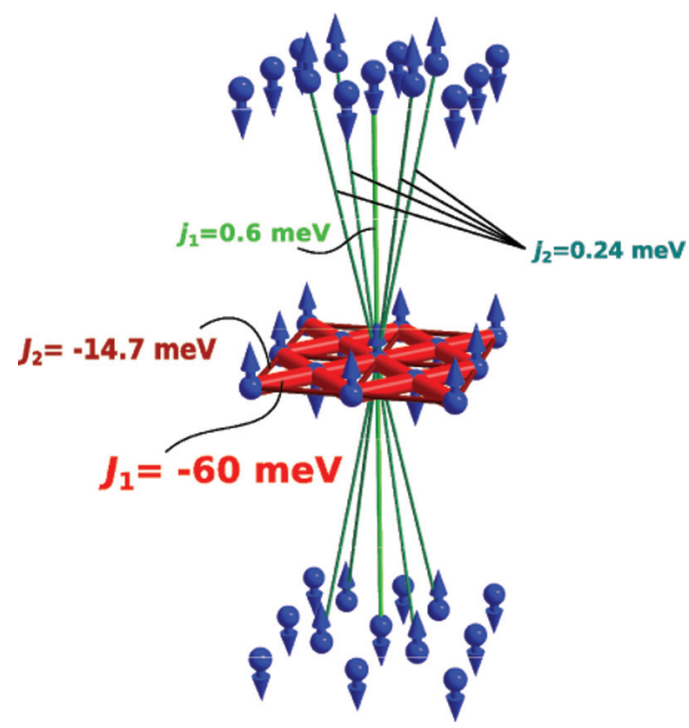

FIG. 16. (Color online) The scheme of the leading magnetic exchange interactions within the Mn atomic planes and between the nearest Mn atomic planes of LiMnAs. For clarity, only Mn atoms are shown (blue spheres) and the orientations of the magnetic moments are depicted with arrows. The thickness of the bonds connecting interacting atoms are roughly proportional to the strength of the corresponding exchange interactions, where antiferromagnetic and ferromagnetic coupling is represented by red $\left(J_{1,2}\right)$ and green $\left(j_{1,2}\right)$ colors, respectively. 
again leads to an overall domination of the next-nearest interplane neighbor coupling $\left(8\left|j_{2}\right|>2 j_{1}\right)$, and in turn leads to an overall ferromagnetic coupling of the adjacent atomic planes.

Based on the Mulliken analysis, the corresponding partial charges, i.e., oxidation numbers of $\mathrm{Li}, \mathrm{Mn}$, and $\mathrm{As}$, were computed as $+0.8,+1.5$, and -2.3 , respectively. This is again in good agreement with the XANES measurement presented above, where an oxidation state of 1.6 was determined for the Mn atom in LiMnAs. For LiMnAs, in our calculations we found an indirect band gap of $0.55 \mathrm{eV}$ [see Fig. 17(a)]. These results are in a good agreement with the recent full potential calculations for bulk LiMnAs, where within the local density approximation (LDA) a $\Gamma$-M indirect gap of approximately 0.5 $\mathrm{eV}^{6}$ was computed. Since it is well known that DFT using LDA or the generalized gradient approximation (GGA, PBE in our case) often underestimates the band gap of semiconductors, instead of commenting on the absolute values we will compare the results of our calculations to the general trends shown by the experimental data.
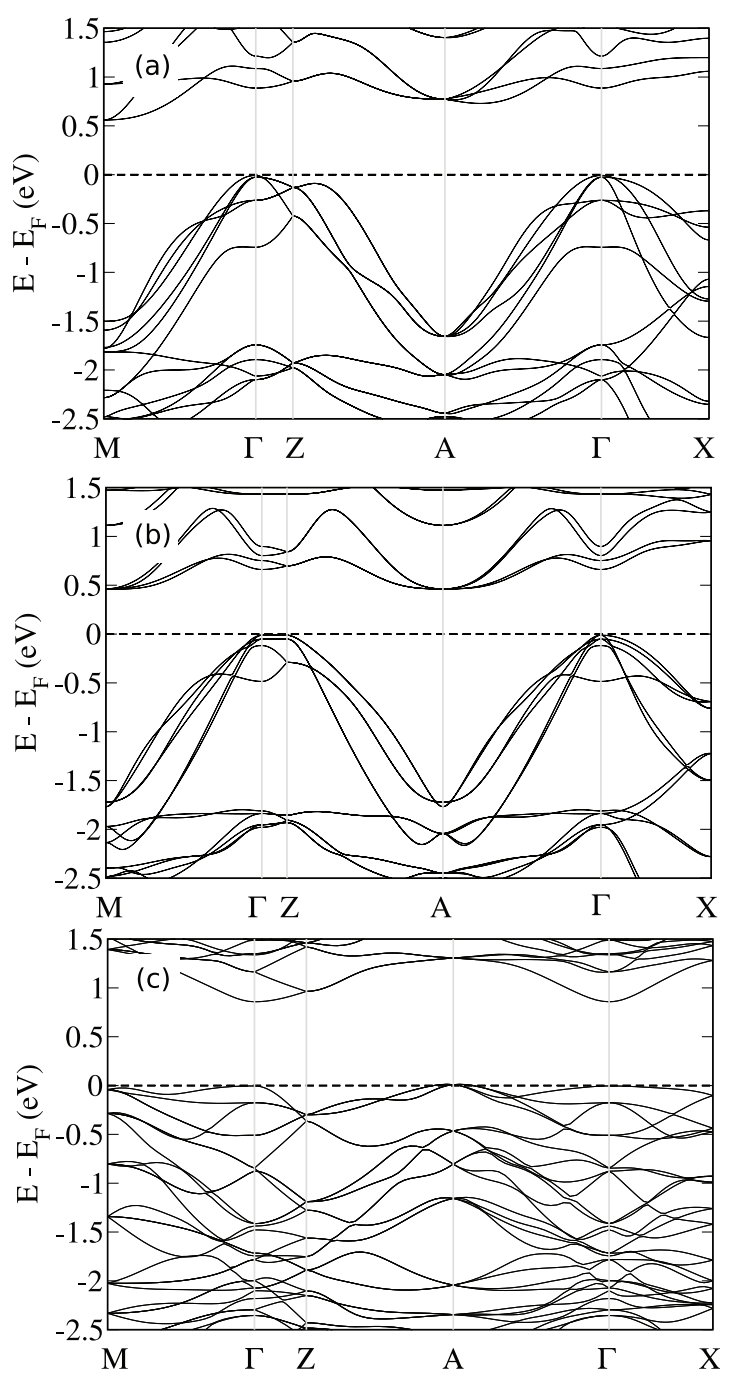

FIG. 17. Electronic band structure of tetragonal antiferromagnetic LiMnAs (a) and LaOMnAs (b). In (c) the band structure of the cubic phase of LiMnAs is shown. Spin-orbit coupling was not taken into account.
As already shown, at $768 \mathrm{~K} \mathrm{LiMnAs} \mathrm{undergoes} \mathrm{a} \mathrm{phase}$ transition to the cubic crystal system. Due to computational limitations and difficulties from the perspective of the methodology, the implicit treatment of temperature effects in ab initio calculations is not straightforward. Therefore, for the study of the cubic phase we have assumed, that the Mn atoms are ordered antiferromagnetically as in the tetragonal phase. Using this model, our calculations with PBE GGA functional indicate that in contrast to the tetragonal phase, the cubic structure has a direct band gap of $0.84 \mathrm{eV}$. For comparison, the experimentally measured electric resistivity data shown in the previous section show the same tendency, namely that the hight temperature cubic phase has a wider band gap.

By comparing the computed total energies of the tetragonal and cubic phases, we found that the cubic phase is only $24 \mathrm{meV}$ higher in energy per formula unit than the tetragonal phase. Thus, although the tetragonal structure is the ground state, the tetragonal-to-cubic phase transition can easily take place. The $24 \mathrm{meV}$ energy difference refers to the difference between the total energies of the static configurations computed for the cubic and tetragonal phase. Thus, this energy difference does not correspond to the activation energy of the phase transition, but it is merely the energy difference between the reactant (tetragonal phase) and product state (cubic phase). Since the calculations are performed for static configurations using the experimental cell parameters and atomic positions at $0 \mathrm{~K}$, they do not contain any information regarding the potential energy landscape, or free-energy contributions. It is out of the scope of the current paper to theoretically describe the reaction mechanism and the underlying reaction barriers involved along the subsequent chemical transformations (i.e., breaking and making of bonds) tracing the corresponding path. Nevertheless, there is good agreement between the theoretically estimated energy difference and the experimentally measured $23 \mathrm{meV} /$ f.u. change in enthalpy, which justifies the use of the simplified theoretical model. Our calculations suggest that AFM ordering dominates in LiMnAs so strongly, to the extent that even if LiMnAs could be synthesized in the cubic phase, the pure undoped compound would still be an AFM semiconductor (at least in the limit of $0 \mathrm{~K}$, to which our calculations refer).

In the case of LaOMnAs, the theoretical local moments as obtained with the PBE functional for the $\mathrm{Mn}$ atoms are $3.54 \mu_{\mathrm{B}}$. In contrast to these results, via neutron diffraction measurements performed at $290 \mathrm{~K}$, the refined magnetic moment of Mn was $2.43 \mu_{\mathrm{B}}$, which is more than one $\mu_{\mathrm{B}}$ lower compared to our theoretical result. In a later experiment, ${ }^{21}$ however, it was shown that the moments at $2 \mathrm{~K}$ are $3.34 \mu_{\mathrm{B}}$, which is in good agreement with our calculations. In the case of LiMnAs, our neutron-diffraction data also show a similar trend as a function of temperature, where at $300 \mathrm{~K}$ the Mn moments are reduced from 3.72 to $2.62 \mu_{\mathrm{B}}$ (see Table I). Furthermore, in the case of the structurally closely related $\mathrm{BaMn}_{2} \mathrm{As}_{2}$ the Mn local moments were determined ${ }^{44}$ to be $3.88 \mu_{\mathrm{B}}$ at 10 $\mathrm{K}$. Hence, the magnetic properties and magnetic moments are rather similar in the whole family of the $A \mathrm{MnAs}(A=\mathrm{Li}$, $\mathrm{LaO}, \mathrm{Ba}$, etc.) layered structure type.

The computed oxidation states of the $\mathrm{La}, \mathrm{O}, \mathrm{Mn}$, and As atoms are $+2.4,-0.8,+1.4$, and -3.1 , respectively; i.e., the oxidation state of $\mathrm{O}$ is underestimated, and that of As is 
overestimated. Regarding the electronic structure, we found that $\mathrm{LaOMnAs}$ is also an indirect band-gap semiconductor with an $E_{\mathrm{g}}$ of $0.46 \mathrm{eV}$ [see Fig. 17(b)]. Thus, in contrast to the experimental results, the theoretically computed values with PBE would suggest that the band gap of LiMnAs is wider than that of LaOMnAs.

\section{CONCLUSION}

In summary, in this paper, we have presented a broad overview of the synthesis, atomic and electronic structure, and magnetic properties of LiMnAs, LaOMnAs, and other related compounds in the $A \mathrm{Mn} X$ family. Thus, with this work, we respond to several important questions raised concerning this important class of materials. In general, the compounds in the $A M n X$ family can be classified into two main categories: AFM semiconductors and FM metals. The nature of the magnetic coupling and of the semiconducting or metallic behavior is mainly determined by the shortest Mn-Mn distances $\left(d_{\mathrm{Mn}-\mathrm{Mn}}\right)$, which seems to be a general property of the compounds containing MnAs layered networks.

For LiMnAs and LaOMnAs, our data are in good agreement with the literature, showing that both are antiferromagnetic semiconductors, with a magnetic moment of about $4 \mu_{\mathrm{B}}$ per $\mathrm{Mn}$ atom at low temperatures. Interestingly, by combining thermal analysis and temperature-dependent XRD measurements, a phase transition to the cubic half-Heusler phase has been discovered. Theoretical calculations suggest that even if cubic LiMnAs could be stabilized, the ideal undoped cubic counterpart would still be an AFM semiconductor, similar to the tetragonal compound. EXAFS and XANES measurements indicate the oxidation state of $\mathrm{Mn}$ to be 1.6. This is in agreement with the concept of localized moments on $\mathrm{Mn}$ and explains the close relationship between the cubic and tetragonal phase and the connection between $\mathrm{Mn}$ and rare-earth ions in tetragonally coordinated structures. Regarding the weak ferromagnetism, in contrast to Refs. 9 and 21, due to symmetry considerations, we expect that canting takes place at the surfaces and interfaces of LiMnAs and LaOMnAs, but in the bulk it is unlikely. Resistivity measurements on LiMnAs and LaOMnAs show two distinct regions of activated transport behavior, and a large change in resistivity by more than five and two orders of magnitude, respectively.

\section{ACKNOWLEDGMENTS}

Financial support from the ERC Advanced Grant (291472) is gratefully acknowledged. We are grateful to Susann Scharsach for the DSC measurements, Dr. Gudrun Auffermann for the chemical analysis measurements, and Igor Veremchuk for the spark plasma sintering (SPS) measurements from the Max Planck Institute for Chemical Physics of Solids. Prof. F. Bernardi received a research grant from $\mathrm{CNPq}$, Brazil. Financial support from DFG Project No. FOR 1464 ASPIMATT(1.2-A) is gratefully acknowledged. We also thank Y.-L. Mathis for providing support at the infrared beamline of the synchrotron facility, ANKA, at the Karlsruhe Institute of Technology. *felser@cpfs.mpg.de

${ }^{1}$ I. Žutić, J. Fabian, and S. Das Sarma, Rev. Mod. Phys. 76, 323 (2004).

${ }^{2}$ T. Dietl, H. Ohno, F. Matsukura, J. Cibert, and D. Ferrand, Science 287, 1019 (2000).

${ }^{3}$ T. Jungwirth, J. Sinova, J. Mašek, J. Kučera, and A. MacDonald, Rev. Mod. Phys. 78, 809 (2006).

${ }^{4}$ Z. Deng, C. Jin, Q. Liu, X. Wang, J. Zhu, S. Feng, L. Chen, R. Yu, C. Arguello, T. Goko, F. Ning, J. Zhang, Y. Wang, A. A. Aczel, T. Munsie, T. Williams, G. Luke, T. Kakeshita, S. Uchida, W. Higemoto, T. Ito, B. Gu, S. Maekawa, G. Morris, and Y. Uemura, Nat. Commun. 2, 422 (2011).

${ }^{5}$ J. Masek, J. Kudrnovský, F. Máca, B. L. Gallagher, R. P. Campion, D. H. Gregory, and T. Jungwirth, Phys. Rev. Lett. 98, 067202 (2007).

${ }^{6}$ T. Jungwirth, V. Novák, X. Martí, M. Cukr, F. Máca, A. B. Shick, J. Mašek, P. Horodyská, P. Němec, V. Holý, J. Zemek, P. Kužel, I. Němec, B. L. Gallagher, R. P. Campion, C. T. Foxon, and J. Wunderlich, Phys. Rev. B 83, 035321 (2011).

${ }^{7}$ V. Novak, M. Cukr, Z. Soban, T. Jungwirth, X. Marti, V. Holy, P. Horodyska, and P. Nemec, J. Cryst. Growth 323, 348 (2011).

${ }^{8}$ A. Wijnheijmer, X. Marti, V. Holy, M. Cukr, V. Novak, T. Jungwirth, and P. Koenraad, Appl. Phys. Lett. 100, 112107 (2012).

${ }^{9}$ N. Emery, E. Wildman, J. Skakle, G. Giriat, R. Smith, and A. Mclaughlin, Chem. Commun. 46, 6777 (2010).
${ }^{10}$ A. Marcinkova, T. C. Hansen, C. Curfs, S. Margadonna, and J. W. G. Bos, Phys. Rev. B 82, 174438 (2010).

${ }^{11}$ E. J. Wildman, J. M. Skakle, N. Emery, and A. C. Mclaughlin, J. Am. Chem. Soc. 134, 8766 (2012).

${ }^{12}$ F. Máca, J. Mašek, O. Stelmakhovych, X. Martí, H. Reichlová, K. Uhlîrová, P. Beran, P. Wadley, V. Novák, and T. Jungwirth, J. Magn. Magn. Mater. 324, 1606 (2012).

${ }^{13}$ J. Wunderlich, T. Jungwirth, B. Kaestner, A. C. Irvine, A. B. Shick, N. Stone, K.-Y. Wang, U. Rana, A. D. Giddings, C. T. Foxon, R. P. Campion, D. A. Williams, and B. L. Gallagher, Phys. Rev. Lett. 97, 077201 (2006)

${ }^{14}$ J. H. Tapp, Z. Tang, B. Lv, K. Sasmal, B. Lorenz, P. C. W. Chu, and A. M. Guloy, Phys. Rev. B 78, 060505R (2008).

${ }^{15}$ W. Bronger, P. Müller, R. Höppner, and H. Schuster, Z. Anorg. Allg. Chem. 539, 175 (1986).

${ }^{16}$ T. Graf, C. Felser, and S. Parkin, Prog. Solid State Chem. 39, 1 (2011).

${ }^{17}$ J. Kübler, A. R. Williams, and C. B. Sommers, Phys. Rev. B 28 , 1745 (1983).

${ }^{18}$ H. Kandpal, C. Felser, and R. Seshadri, J. Phys. D: Appl. Phys. 39, 776 (2006)

${ }^{19}$ C. Felser, G. Fecher, and B. Balke, Angew. Chem. Int. Ed. 46, 668 (2007).

${ }^{20}$ F. Schucht, A. Dascoulidou, R. Müller, W. Jung, H.-U. Schuster, W. Bronger, and P. Müller, Z. Anorg. Allg. Chem. 625, 31 (1999). 
${ }^{21}$ N. Emery, E. J. Wildman, J. M. S. Skakle, A. C. Mclaughlin, R. I. Smith, and A. N. Fitch, Phys. Rev. B 83, 144429 (2011).

${ }^{22}$ R. Müller, M. Kuckel, H.-U. Schuster, P. Müller, and W. Bronger, J. Alloy. Compd. 176, 167 (1991).

${ }^{23}$ M. Otto, H. Feil, R. A. M. van Woerden, J. Wijngaard, P. Van Der Valk, C. F. van Bruggen, and C. Haas, J. Magn. Magn. Mater. 70, 33 (1987).

${ }^{24}$ V. Dinh, K. Sato, and H. Katayama-Yoshida, J. Phys. Soc. Jpn. 77, 014705 (2008).

${ }^{25}$ P. Van Engelen, D. De Mooij, J. Wijngaard, and K. Buschow, J. Magn. Magn. Mater. 130, 247 (1994).

${ }^{26}$ F. Ishikawa, K. Koyama, K. Watanabe, T. Asano, and H. Wada, J. Phys. Soc. Jpn. 75, 084604 (2006).

${ }^{27}$ S. Haneda, N. Kazama, Y. Yamaguchi, and H. Watanabe, J. Phys. Soc. Jpn. 42, 1201 (1977).

${ }^{28}$ J. W. Simonson, Z. P. Yin, M. Pezzoli, J. Guo, J. Liu, K. Post, A. Efimenko, N. Hollmann, Z. Hu, H.-J. Lin, C.-T. Chen, C. Marques, V. Leyva, G. Smith, J. W. Lynn, L. L. Sun, G. Kotliar, D. N. Basov, L. H. Tjeng, and M. C. Aronson, Proc. Natl. Acad. Sci. USA 109, 10751 (2012).

${ }^{29}$ G. Achenbach and H. U. Schuster, Z. Anorg. Allg. Chem. 9, 475 (1981).

${ }^{30}$ V. Petricek, M. Dusek, and L. Palatinus, Jana 2006, the Crystallographic Computing System (Institute of Physics, Praha, Czech Republic, 2006).

${ }^{31}$ I. E. Dzyaloshinskii, J. Chem. Solids 4, 241 (1958).
${ }^{32}$ T. Moriya, Phys. Rev. Lett. 4, 228 (1960).

${ }^{33}$ D. Konigsberger and R. Prins, editors, X-Ray Absorption: Principles, Applications, and Techniques of EXAFS, SEXAFS, and XANES, Chemical Analysis, Vol. 92 (Wiley, New York, 1988).

${ }^{34}$ M. Newville, J. Synchrotron Radiat. 8, 322 (2001).

${ }^{35}$ S. I. Zabinsky, J. J. Rehr, A. Ankudinkov, R. C. Albers, and M. J. Eller, Phys. Rev. B 52, 2995 (1995).

${ }^{36}$ J. Wong, F. W. Lytle, R. P. Messmev, and D. H. Maylotte, Phys. Rev. B 30, 5596 (1984).

${ }^{37}$ C. Kittel, Introduction to Solid State Physics, 4th ed. (Wiley, New York, 1973).

${ }^{38}$ Spectroscopic Ellipsometry Data Acquisition and Analysis Software WVASE32, J. A. Woollam Co., Inc., http://www.jawoollam.com.

${ }^{39}$ A. V. Boris, N. N. Kovalena, S. S. A. Seo, J. S. Kim, P. Popovich, Y. Matiks, R. K. Kremer, and B. Keimer, Phys. Rev. Lett. 102, 027001 (2009).

${ }^{40}$ G. Kresse and J. Furthmüller, Phys. Rev. B 54, 11169 (1996).

${ }^{41}$ G. Kresse and J. Furthmüller, Comput. Mater. Sci. 6, 15 (1996).

${ }^{42}$ J. P. Perdew, K. Burke, and M. Ernzerhof, Phys. Rev. Lett. 77, 3865 (1996).

${ }^{43}$ H. Ebert, D. Ködderitzsch, and J. Minár, Rep. Prog. Phys. 74, 096501 (2011)

${ }^{44}$ Y. Singh, M. A. Green, Q. Huang, A. Kreyssig, R. J. McQueeney, D. C. Johnston, and A. I. Goldman, Phys. Rev. B 80, 100403 (2009). 